\title{
Nasal self-packing for epistaxis in Hereditary Hemorrhagic Telangiectasia increases quality of life*
}

\author{
Freya Droege1, Carolin Lueb', Kruthika Thangavelu', Boris A. Stuck², \\ Stephan Lang', Urban Geisthoff²
}

'Department of Otorhinolaryngology, Head and Neck Surgery, Essen University Hospital, University Duisburg-Essen, Essen, Germany

2 Department of Otorhinolaryngology, Head and Neck Surgery, University Hospital of Marburg, Philipps-Universität Marburg, Marburg, Germany
Rhinology 57: 3, 231 - 239, 2019

https://doi.org/10.4193/Rhin18.141

*Received for publication:

July 25, 2018

Accepted: January 28, 2019

\begin{abstract}
Statement of problem: Hereditary hemorrhagic telangiectasia (HHT) is characterized by recurrent epistaxis that can lead to a feeling of losing control. We assessed potential benefits and side effects of different nasal packings used by patients themselves.

Method of study: An online-questionnaire in English and German was used to analyze nasal self-packings.

Results: 588 of the 915 respondents suffered from HHT with most of them having moderate or severe epistaxis. Almost two thirds of the patients had already treated themselves with nasal packings. While one quarter used non-pneumatic nasal packings (NPNP) or pneumatic nasal packings (PNP), nearly half of the patients took only tissues to stop the bleeding. Patients with severe epistaxis used PNP more often than NPNP. Using nasal packings, most patients could stop their nosebleeds after a while. Patients using PNP reported the feeling of losing control less often and significant improvements in quality of life with a positive GBI.
\end{abstract}

Conclusions: Our study showed that most patients with HHT using nasal self-packings could stop the bleeding after a while. Nasal self-packing is a user-friendly and secure method leaving patients more self-confident and independent.

Key words: epistaxis, nosebleeds, quality of life, nasal mucosa, nasal obstruction

\section{Introduction}

Hereditary hemorrhagic telangiectasia (HHT) is an inherited disorder leading to systemic vascular malformations. Patients suffer from multiple typical telangiectases and larger visceral vascular malformations especially in the lungs, the liver and brain. Epistaxis is the most frequent manifestation of HHT. Clinical diagnosis of HHT can be established using the Curaçao criteria (1). Mutations in several genes of the transforming growth factor (TGF)- $\beta$ superfamily pathway influence angiogenesis in patients with $\mathrm{HHT}^{(2-6)}$. Recurrent nosebleeds can lead to significant blood loss resulting in anemia and potentially lethal complications such as hypovolemia and shock ${ }^{(7)}$. Besides, the physical impairment, patients experience a feeling of 'losing control', since they have limited options to influence the duration and frequency of nosebleed episodes. This may result in a reduction of? their quality of life ${ }^{(8-10)}$. There are different therapeutic approaches for treating recurrent epistaxis. Strategies such as destroying nasal telangiectasia (e.g. with different types of coagulation) or the reduction of endonasal trauma (e.g. with nasal ointments, surgical nasal closure or with different medical devices) may have a preventive effect ${ }^{(11)}$. In the acute event of a severe nosebleed, patients need tools that are readily available and easy to handle. Over 100 years ago, Sir W. Osler described a patient stopping the bleeding by inserting and inflating a rubber arrangement inside his nose ${ }^{(12)}$. Nowadays, a range of different non degradable, inflatable and absorbable nasal packings are used ${ }^{(13)}$ to prevent severe nasal blood loss. HHT as such, however, remains an incurable disease.

The main aim of this study was to assess the practices of HHT patients when self-administering nasal packings and the resulting effect on patients' lives. A multitude of parameters including the type of packing, severity of nosebleeds, hemoglobin level, iron intake, patients' safety and comfort were documented. 


\section{Material and methods}

Compliance with ethical standards

All procedures performed in studies involving human participants were in accordance with the 1964 Helsinki declaration (or Declaration of Helsinki) and its later amendments or comparable ethical standards. The study was approved by the Ethics Committee of the University Duisburg-Essen (15-6429-BO). Data were provided voluntarily by the patients.

\section{Study design and setting}

A questionnaire was developed in English and German and published online. One otorhinolaryngologist and one patient with $\mathrm{HHT}$, both of whom were native English speakers and fluently bilingual in English and German translated the questionnaire. Afterwards, all other authors and a German-speaking HHT patient crosschecked the translation and optimized it in collaboration with the two native speakers.

The survey was disseminated through different patient advocacy groups all over the world (see acknowledgments). The survey included the following sections:

$\begin{array}{ll}- & \text { General medical history of HHT } \\ \text { - } & \text { Epistaxis Severity Score (ESS) for HHT } \\ \text { - } & \text { Treatment of epistaxis } \\ \text { - } & \text { Nasal self-packings } \\ \text { - } & \text { Glasgow Benefit Inventory (GBI) }\end{array}$

The general medical history of HHT contained questions about the Curaçao criteria ${ }^{(1)}$ and results of a possible genetic testing. The ESS for $\mathrm{HHT}^{(14)}$ had questions on the frequency, duration, and intensity of epistaxis. In addition, the need for medical attention, transfusions related to epistaxis, and signs of anemia (hemoglobin levels) were documented. Respondents were asked to describe their acute and preventive treatments of recurrent nosebleeds. Patients who administered their own nasal self-packings were divided in two categories: those using only tissues and those that use medical nasal packings. The latter were divided further into those also inserting pneumatic nasal packings (PNP, this group includes patients using PNP and patients using both NPNP and PNP) and those only using nonpneumatic nasal packings (NPNP) (Figure 1). The benefits and complications of each type of nasal self-packing were documented. Validated English and German versions of the GBI were used to detect the influence of nasal self-packing on the quality of life. The $\mathrm{GBI}$ is an 18-item questionnaire measuring the resulting benefit of especially otorhinolaryngological interventions ${ }^{(15)}$.

\section{Outcome variables and statistical analysis}

Epistaxis was classified using the ESS ${ }^{(14)}$. Women with an average hemoglobin level below $12.0 \mathrm{~g} / \mathrm{dl}$ and men with a level below $13.0 \mathrm{~g} / \mathrm{dl}$ were classified as having anemia ${ }^{(16)}$. Patients with $\mathrm{HHT}$ reported the effectiveness of stopping the bleeding of the nasal packing on a scale of 1 to 5 , where 1 was "bleeding

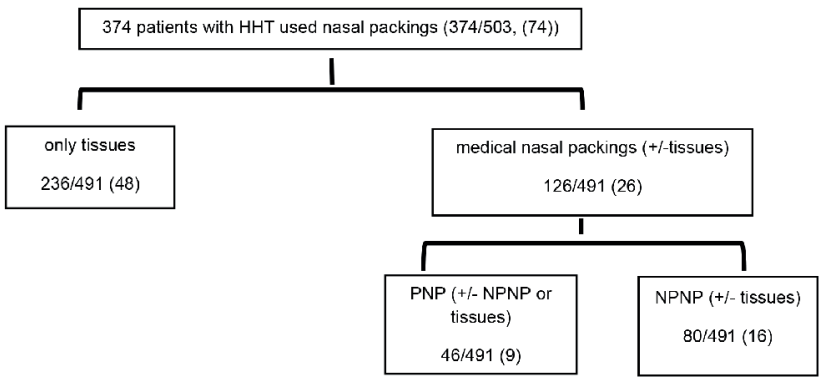

Figure 1. Categorization of nasal self-packings. 374 patients with HHT (out of 503 patients who answered the question if they have used any kind of nasal selfpackings so far) administered their own nasal selfpackings. In another question patients were asked to specify the used nasal self-packing. 491 patients with HHT answered this question. 129 patients stated that they have not used any kind of nasal packing $(\mathrm{N}=$ 129/491, 26\%). The other 362 patients ( $N=362 / 491,74 \%$ ) were divided in two categories: those taking only tissues and those also using medical nasal packings ( \pm tissues). The latter were divided further info those also inserting pneumatic nasal packings (PNP, this includes patients using only PNP and those inserting PNP \pm NPNP \pm tissues) and those using non-pneumatic nasal packings (NPNP \pm tissues). Each patient was categorized in a single group. Data is presented in number of patients $(\mathrm{N})$ and percentage (\%).

continued" and 5 was "bleeding stopped immediately". Patients with recurrent epistaxis also recorded the feeling of losing control of the situation on a scale of 1 to 5 , with 1 being "very often" and 5 "never".

Descriptive statistics (number/ percentage of patients $(\mathrm{N}, \%)$ and mean \pm standard deviation $(\mathrm{m} \pm \mathrm{SD})$ ) were used for the general history of HHT and clinical presentations of epistaxis. Different patient groups were compared using two-sample t-tests, oneway ANOVA, Kruskal-Wallis-tests and chi-square test of differences. Statistical analyses were performed with IBM SPSS Statistics (version 25, Armonk, NY: IBM Corp. Released 2017).

\section{Results}

Clinical presentation of patients with HHT In 588 of 915 survey respondents (64\%), the diagnosis of HHT could be established using the criteria published elsewhere (17). For another 15 participants ( $\mathrm{N}=15 / 915,2 \%)$, a diagnosis of HHT could not be assigned with complete confidence. Most of the respondents were patients ( $\mathrm{N}=553 / 582,95 \%)$, while relatives answered the questionnaire for a patient in $5 \%$ of the cases ( $N$ $=29 / 582$ ). The following data refers to the 588 patients with a confirmed diagnosis of HHT. Not all patients with $\mathrm{HHT}$ answered all questions completely, and all numbers in the results are given in relation to sufficiently answered questions (Figure 2 ). About two thirds of the respondents were female (female: $\mathrm{N}=$ $326 / 477,68 \%$; male: $N=151 / 477,32 \%)$, and they were signifi- 


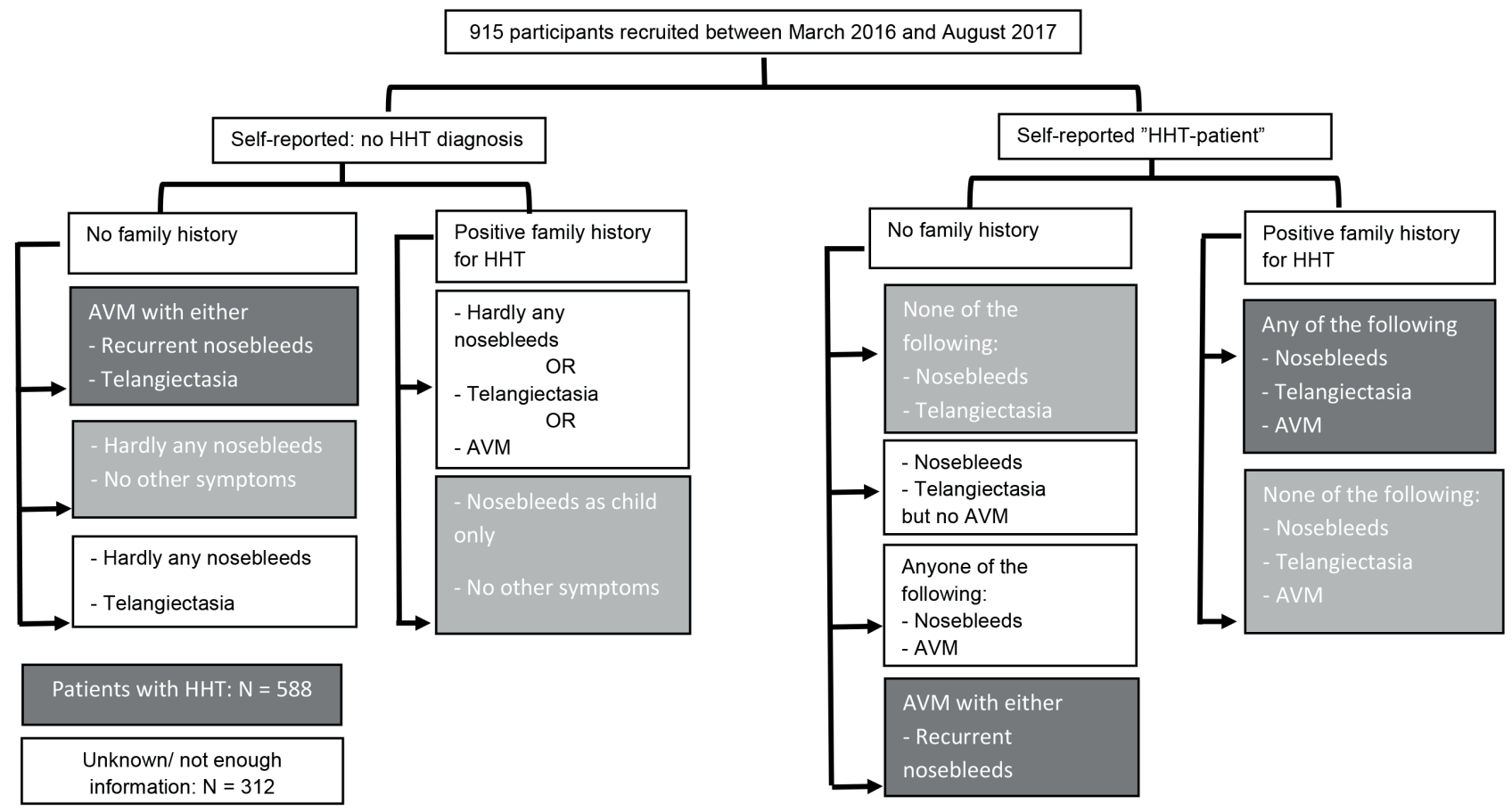

Figure 2. Stratification of diagnostic assignments. Using the Curaçao criteria patients with first degree relative with HHTI positive family history, spontaneous and recurrent epistaxis, multiple teleangiectasia at characteristical sites and/or visceral ateriovenous malfromations (AVMs) or telangiectases (e.g. cerebral, pulmonary, hepatic or gastrointestinal involvement) are documented. Patients fulfilling two out of these four criteria are categorized as probable HHT patients and patients fulfilling three or four criteria are diagnosed as HHT patients ${ }^{(1)}$. Similarly to Hosman et al. ${ }^{(17)}$, this flowchart shows the modified Curaçao criteria white using different terms to describe the three groups "Patients with HHT", "unknown not enough information" and "persons without HHT". HHT = hereditary hemorrhagic telangiectasia, AVM = ateriovenous malformation (e.g. hepatic cerebral pulmonary vascular malformation or gastrointestinal involvement), $\mathrm{N}=$ number of paticipants.

cantly younger than men (female: average age $=54 \pm 11$ years, $\mathrm{N}=104 / 150$; male: average age $=60 \pm 13$ years, $\mathrm{N}=46 / 150 ; \mathrm{T}-$ Test $=-2.97, \mathrm{p}=0.004)$. HHT patients all over the world reported using nasal packings (North America: $\mathrm{N}=187$, Western Europe: $\mathrm{N}=40$, Australia: $\mathrm{N}=17$, North Africa: $N=7$, Eastern Europe: $N$ $=2$, South America: $\mathrm{N}=2$, Asia: $\mathrm{N}=2$, Middle East: $\mathrm{N}=1$, Central America: $\mathrm{N}=1$ ).

Over three quarters (77\%) of the respondents stated a general progression of disease $(\mathrm{N}=450 / 586)$, and $18 \%(\mathrm{~N}=106 / 586)$ had a stable disease. Only $5 \%(\mathrm{~N}=30 / 586)$ reported a general improvement of the disease. Almost all respondents experienced recurrent epistaxis and multiple typical telangiectases (Table 1). The respondents had an average hemoglobin level of $10.6 \mathrm{~g} / \mathrm{dl}(\mathrm{SD} \pm 2.7)$ with $84 \%(\mathrm{~N}=431 / 514)$ of the HHT-patients stated suffering from anemia. Female and male patients were equally affected by anemia (t-test $=1.27, p=0.205)$. Most patients took iron preparations ( $\mathrm{N}=480 / 577,83 \%$; iron tablets/ juice: $N=258 / 480,54 \%$; infusions/injections: $N=47 / 480,10 \%$; tablets and infusions: $\mathrm{N}=175 / 480,37 \%)$. Thirty-eight per cent of HHT patients $(\mathrm{N}=168 / 437)$ reported that they received at least one blood transfusion for bleeding-associated anemia (average number of blood transfusions $=23 \pm 85$ ).

\section{Treatment of epistaxis}

HHT patients stated that their nosebleeds began at an average age of 16 years ( $S D=12$ years), and $87 \%(N=461 / 531)$ had consulted a doctor for treating the nosebleeds. Men had a significant higher consultation rate than women (consultation rate in women: $265 / 312,85 \%$; in men: $132 / 144,92 \% ; \chi^{2}: 3.962, p=$ 0.047). Using the Epistaxis Severity Score (ESS), $19 \%(N=62 / 331$ ) of the patients with HHT suffered from mild nosebleeds; $48 \%$ (N $=158 / 331)$ had moderate nosebleeds; and 34\% ( $N=111 / 331)$ had severe nose bleedings. Men and women were equally affected in our sample. Older patients showed a tendency to suffer more often from severe bleedings (F-value: $2.386 ; p=0.098$ ) and had a higher consultation rate than younger patients (t-test: -8.227; $\mathrm{p}$ <.001). In a multivariate logistic regression model, ESS had a significant association with the patients' consultation rate $(\mathrm{OR}=1.5,95 \% \mathrm{Cl} 1.0-2.1, \mathrm{p}=0.02)$. Patients with severe epistaxis had significantly lower hemoglobin levels than those 
Table 1. Different manifestations of HHT according to the Curaçao Criteria.

\begin{tabular}{|c|c|c|c|c|c|}
\hline & & & $\begin{array}{c}\text { all } \\
\text { patients } \\
\text { ( } \mathbf{N}=\mathbf{5 8 8})\end{array}$ & $\begin{array}{l}\text { women } \\
(\mathrm{N}=\mathbf{3 2 6})\end{array}$ & $\begin{array}{c}\text { men } \\
(N=151)\end{array}$ \\
\hline & & yes & 550 (93.7) & 310 (95.4) & 135 (89.4) \\
\hline & & no & 19 (3.2) & $7(2.2)$ & $10(6.6)$ \\
\hline Taminy & sistory & n.k. & $18(3.1)$ & $8(2.5)$ & $6(4.0)$ \\
\hline & & $\mathrm{mv}$ & 1 & 1 & 0 \\
\hline & & yes & 532 (96.6) & 309 (95.1) & $146(98.0)$ \\
\hline epi & xis & no & $19(3.4)$ & $16(4.9)$ & $3(2.0)$ \\
\hline & & $\mathrm{mv}$ & 37 & 1 & 2 \\
\hline & & yes & $557(96.4)$ & 309 (95.1) & $146(97.3)$ \\
\hline & & no & $14(2.4)$ & $10(3.1)$ & $4(2.7)$ \\
\hline & & n.k. & $/(1.2)$ & $6(1.8)$ & 0 \\
\hline & & $\mathrm{mv}$ & 10 & 1 & 1 \\
\hline & & yes & 191 (35.5) & $108(34.8)$ & $55(38.7)$ \\
\hline & GI & no & 159 (29.6) & $88(28.4)$ & 45 (31.7) \\
\hline & U & n.k. & 188 (34.9) & $114(36.8)$ & $42(29.6)$ \\
\hline & & $\mathrm{mv}$ & 50 & 16 & 9 \\
\hline & & yes & 251 (45.6) & $146(45.9)$ & 61 (42.7) \\
\hline & PAVM & no & $193(35.0)$ & 107 (33.6) & 57 (39.9) \\
\hline & PAVIVI & n.k. & $107(19.4)$ & 65 (20.4) & 25 (17.5) \\
\hline Visceral & & mv & 37 & 8 & 8 \\
\hline lesions & & yes & $135(25.5)$ & $79(25.8)$ & 26 (18.6) \\
\hline & HVM & no & 187 (35.3) & $101(33.0)$ & 64 (45.7) \\
\hline & invivi & n.k. & 207 (39.1) & $126(41.29)$ & $50(35.7)$ \\
\hline & & $\mathrm{mv}$ & 59 & 20 & 11 \\
\hline & & yes & 73 (13.6) & 42 (13.6) & 19 (13.4) \\
\hline & CVM & no & $325(60.4)$ & $182(58.9)$ & 87 (61.3) \\
\hline & Cvivi & n.k. & $140(26.0)$ & 85 (27.5) & $36(25.4)$ \\
\hline & & $\mathrm{mv}$ & 50 & 17 & 9 \\
\hline
\end{tabular}

$\mathrm{HHT}=$ hereditary hemorrhagic telangiectasia, $\mathrm{TAE}=$ telangiectasia, $\mathrm{GI}=$ gastrointestinal involvement, PAVM = pulmonary arteriovenous malformation, HVM = hepatic vascular malformation, $C V M=$ cerebral vascular malformation, $\mathrm{N}=$ number of patients, $\mathrm{n} . \mathrm{k}$. = not known, $\mathrm{mv}=$ missing values. Data are presented as N (\%) (percentages are calculated after exclusion of missing values). Gender was not documented in all cases.

with mild or moderate epistaxis (hemoglobin levels - severe epistaxis: $9.7 \mathrm{~g} / \mathrm{dl}$, moderate epistaxis: $11.1 \mathrm{~g} / \mathrm{dl}$, mild epistaxis: $12.5 \mathrm{~g} / \mathrm{dl}$; F-value: $21.3 ; \mathrm{p}<0.000)$.

\section{Nasal self-packing}

Most patients with HHT knew about the possibility of nasal self-packings ( $N=460 / 503,92 \%)$, and 33\% ( $N=158 / 476)$ knew others who helped themselves with nasal packings. Nearly two thirds had treated their epistaxis with nasal packings $(\mathrm{N}=$ $374 / 503,74 \%)$. Almost half (46\%) of the patients inserting medical packings $(\mathrm{N}=58 / 125)$ were trained by their otorhinolaryngologist and one patient (1\%) had learned it from his/her hematologist. About one quarter ( $N=29 / 125,23 \%)$ were self-taught. Twenty-three patients (18\%) saw YouTube instructional videos and 13 patients (10\%) learned it from their family or friends. One patient (1\%) was taught by a HHT patient association/ self-help group.

Four hundred and ninety-one patients answered the question about the exact type of nasal self-packing patients used. Of these, one quarter had used medical packings ( $N=126 / 491$, $26 \%$ ) and almost half of all patients used only tissues to stop the bleeding ( $N=236 / 491,48 \%$ ). Many patients used more than one type of nasal packing. Eighty of them used only non-pneumatic nasal packings (NPNP) to treat nasal bleedings ( $N=80 / 491$, $16 \%)$. Almost every tenth patient with HHT ( $\mathrm{N}=46 / 491,9 \%)$ also inserted PNP (this group of patients included patients inserting PNP and those using both PNP and NPNP, Table 2). Patients using no packings and those using NPNP had the lowest ESS (F-value: 6.014, p = 0.001). In contrast, patients also using PNP reported that they suffered from severe epistaxis $\left(\chi^{2}: 15.945, p\right.$ $=0.014$ ) and needed more blood transfusions (F-value: $3.785, \mathrm{p}$ $=0.011$ ). There was no significant difference in the hemoglobin levels of patients with or without nasal packings (F-value: 1.785, $\mathrm{p}=0.150)$

Eighty-six patients with HHT using nasal self-packings ( $\mathrm{N}=$ $86 / 347,25 \%$ ) reported complications or difficulties due to selfpacking and 31 ( $N=31 / 312,10 \%)$ knew other people who had experienced difficulties or complications. The main complaint among all types of packings was the time spent in stopping the epistaxis sufficiently ( $N=24 / 86,28 \%)$. Among the different types of nasal packings, patients using PNP felt significantly more pain ( $x 2: 6.826, p=0.033)$. However, patients using only tissues or NPNP more often complained of swallowing parts of it $\left(\chi^{2}\right.$ : $6.886, p=0.032$ ). There was no significant difference in number and severity of reported complications based on how patients learned to use nasal packings (Table 3).

Both patient groups using nasal packings and those who did not use any packing reported that the bleeding stopped after a while (median = scale of 4 , Kruskal Wallis test: $p=0.278$ ). In general, patients had the feeling of losing control less frequently after learning to administer nasal self-packing. In particular, patients inserting PNP experienced feelings of helplessness significantly less often (median of differences between the feeling of losing control before and after learning how to use nasal packings $=1$; Kruskal Wallis test: $\mathrm{p}=0.016$ )

Patients with PNP gained the highest score in the GBI compared to those taking NPNP and those using only tissues (F-value: 6.981, $p=0.001)$. Patients inserting PNP achieved the highest scores for the general and physical sub-scales (general subscale: F-value: 7.747, $p=0.001$, physical: F-value: $2.734, p=0.067$ ). However, for the social subscale, patients using only tissues reached the highest score (F-value: 0.495, $p=0.610$ ) (Table 4).

\section{Discussion}

Epistaxis is a cardinal symptom in patients with HHT with more than $90 \%$ suffering from recurrent nosebleeds ${ }^{(18)}$. An ideal nasal 
Table 2. Epistaxis data of patients using no or different types of nasal packings.

\begin{tabular}{|c|c|c|c|c|c|c|c|}
\hline & & & $\operatorname{sex}$ & $\begin{array}{l}\text { frequency } \\
\text { [N (\%)] }\end{array}$ & $\begin{array}{l}\text { hb level } \\
{[m \pm S D]}\end{array}$ & $\begin{array}{c}\text { BT } \\
{[m \pm S D]}\end{array}$ & $\begin{array}{c}\text { ESS } \\
{[m \pm S D]}\end{array}$ \\
\hline $\begin{array}{l}\text { no nasal } \\
\text { packing }\end{array}$ & & & $\begin{array}{l}\text { W: } \\
\text { M: } \\
\text { all: }\end{array}$ & $\begin{array}{c}98(31.9) \\
23(16.1) \\
129(26.3)\end{array}$ & $\begin{array}{l}10.5 \pm 2.4 \\
11.7 \pm 2.2 \\
10.8 \pm 2.3\end{array}$ & $\begin{array}{c}27 \pm 47 \\
3 \pm 3 \\
22 \pm 43\end{array}$ & $\begin{array}{l}5.5 \pm 2.1 \\
5.1 \pm 2.3 \\
5.4 \pm 2.1\end{array}$ \\
\hline \multirow[t]{14}{*}{$\begin{array}{l}\text { type of nasal } \\
\text { packing }\end{array}$} & tissue & & $\begin{array}{l}\text { W: } \\
\text { M: } \\
\text { all: }\end{array}$ & $\begin{array}{c}138(45.0) \\
71(49.7) \\
320(85.8)\end{array}$ & $\begin{array}{l}10.6 \pm 2.7 \\
10.1 \pm 2.7 \\
10.4 \pm 3.0\end{array}$ & $\begin{array}{c}9 \pm 13 \\
13 \pm 23 \\
11 \pm 19\end{array}$ & $\begin{array}{l}5.7 \pm 2.1 \\
6.3 \pm 2.1 \\
6.0 \pm 2.1\end{array}$ \\
\hline & PNP & Rapid Rhino ${ }^{\circ}$ & $\begin{array}{l}\text { W: } \\
\text { M: } \\
\text { all: }\end{array}$ & $\begin{array}{l}25(11.7) \\
19(15.4) \\
46(12.3)\end{array}$ & $\begin{array}{l}10.1 \pm 1.5 \\
11.3 \pm 2.8 \\
10.6 \pm 2.2\end{array}$ & $\begin{array}{c}36 \pm 116 \\
40 \pm 77 \\
43 \pm 103\end{array}$ & $\begin{array}{l}7.8 \pm 1.9 \\
7.5 \pm 2.4 \\
7.7 \pm 2.0\end{array}$ \\
\hline & NPNP & $\begin{array}{l}\text { Tabotamp }{ }^{\oplus /} \\
\text { SURGICEL }{ }^{\circledR}\end{array}$ & $\begin{array}{l}\text { W: } \\
\text { M: } \\
\text { all: }\end{array}$ & $\begin{array}{c}13(6.1) \\
18(14.6) \\
33(8.8)\end{array}$ & $\begin{array}{l}11.5 \pm 1.8 \\
11.3 \pm 2.6 \\
11.1 \pm 2.5\end{array}$ & $\begin{array}{l}26 \pm 28 \\
12 \pm 13 \\
16 \pm 20\end{array}$ & $\begin{array}{l}8.0 \pm 1.8 \\
6.5 \pm 1.8 \\
7.2 \pm 1.9\end{array}$ \\
\hline & & Stryphnasal $^{\circledR}$ & $\begin{array}{l}\text { W: } \\
\text { M: } \\
\text { all: }\end{array}$ & $\begin{array}{c}20(9.3) \\
9(7.3) \\
31(8.3)\end{array}$ & $\begin{array}{l}10.7 \pm 3.0 \\
11.3 \pm 2.6 \\
10.9 \pm 2.7\end{array}$ & $\begin{array}{l}19 \pm 23 \\
11 \pm 12 \\
16 \pm 20\end{array}$ & $\begin{array}{c}5.6 \pm 2.8 \\
4.3 \\
6.0 \pm 3.0\end{array}$ \\
\hline & & Nasal CEASE ${ }^{\oplus}$ & $\begin{array}{l}\text { W: } \\
\text { M: } \\
\text { all: }\end{array}$ & $\begin{array}{l}15(7.0) \\
10(8.1) \\
26(7.0)\end{array}$ & $\begin{array}{c}10.9 \pm 2.1 \\
13.2 \pm 2.8 \\
12 \pm 2.6\end{array}$ & $\begin{array}{c}13 \pm 19 \\
7 \pm 4 \\
12 \pm 16\end{array}$ & $\begin{array}{l}5.9 \pm 2.4 \\
5.0 \pm 2.2 \\
5.4 \pm 2.3\end{array}$ \\
\hline & & GELASPON ${ }^{\circledR}$ & $\begin{array}{l}\text { W: } \\
\text { M: } \\
\text { all: }\end{array}$ & $\begin{array}{l}5(2.3) \\
1(0.8) \\
6(1.6)\end{array}$ & $\begin{array}{c}8.1 \pm 3.9 \\
7.5 \\
8.0 \pm 3.4\end{array}$ & $\begin{array}{c}6 \pm 6 \\
- \\
6 \pm 6\end{array}$ & $\begin{array}{c}6.0 \\
- \\
6.0\end{array}$ \\
\hline & & KALTOSTAT ${ }^{\oplus}$ & $\begin{array}{l}\text { W: } \\
\text { M: } \\
\text { all: }\end{array}$ & $\begin{array}{l}3(1.4) \\
2(1.6) \\
5(1.3)\end{array}$ & $\begin{array}{l}10.0 \pm 1.4 \\
13.3 \pm 0.4 \\
11.6 \pm 2.1\end{array}$ & $\begin{array}{c}2 \\
2 \\
2 \pm 0\end{array}$ & $\begin{array}{c}6.0 \pm 3.6 \\
6.7 \\
6.2 \pm 3.0\end{array}$ \\
\hline & & NasoPORE ${ }^{\oplus}$ & $\begin{array}{l}\text { W: } \\
\text { M: } \\
\text { all: }\end{array}$ & $\begin{array}{l}2(0.9) \\
2(1.6) \\
4(1.1)\end{array}$ & $\begin{array}{c}10 \pm 1.4 \\
12.0 \pm 5.7 \\
11.0 \pm 3.6\end{array}$ & $\begin{array}{c}1 \\
7 \\
4 \pm 4\end{array}$ & $\begin{array}{l}- \\
- \\
-\end{array}$ \\
\hline & & Merocel $^{\circledast}$ & $\begin{array}{l}\text { W: } \\
\text { M: } \\
\text { all: }\end{array}$ & $\begin{array}{l}1(0.5) \\
1(0.8) \\
3(0.8)\end{array}$ & $\begin{array}{c}8.5 \\
14.5 \\
10.3 \pm 3.6\end{array}$ & $\begin{array}{c}10 \\
- \\
105 \pm 143\end{array}$ & $\begin{array}{c}- \\
3.6 \\
5.9 \pm 3.3\end{array}$ \\
\hline & & $\begin{array}{c}\text { Kwizda First Aid } \\
\text { Nose Plug }^{\circledR}\end{array}$ & $\begin{array}{l}\text { W: } \\
\text { M: } \\
\text { all: }\end{array}$ & $\begin{array}{l}1(0.5) \\
2(1.6) \\
3(0.8)\end{array}$ & $\begin{array}{c}9.0 \\
11.3 \pm 3.9 \\
10.5 \pm 3.0\end{array}$ & $\begin{array}{c}40 \\
25 \\
33 \pm 11\end{array}$ & $\begin{array}{l}- \\
- \\
-\end{array}$ \\
\hline & & NASASTENT ${ }^{\odot}$ & $\begin{array}{l}\text { W: } \\
\text { M: } \\
\text { all: }\end{array}$ & $\begin{array}{c}1(0.5) \\
0(0) \\
1(0.3)\end{array}$ & $\begin{array}{c}11.0 \\
- \\
11.0\end{array}$ & $\begin{array}{l}1 \\
- \\
1\end{array}$ & $\begin{array}{l}- \\
- \\
-\end{array}$ \\
\hline & & Rhino Clip ${ }^{\oplus}$ & $\begin{array}{l}\text { W: } \\
\text { M: } \\
\text { all: }\end{array}$ & $\begin{array}{c}0(0) \\
1(0.8) \\
1(0.3)\end{array}$ & $\begin{array}{l}- \\
9.0 \\
9.0\end{array}$ & $\begin{array}{l}- \\
8 \\
8\end{array}$ & $\begin{array}{l}- \\
8.7 \\
8.7\end{array}$ \\
\hline & & Ointment strips & $\begin{array}{l}\text { W: } \\
\text { M: } \\
\text { all: }\end{array}$ & $\begin{array}{c}1(0.5) \\
0(0) \\
1(0.3)\end{array}$ & $\begin{array}{c}12.0 \\
- \\
12.0\end{array}$ & $\begin{array}{l}- \\
- \\
-\end{array}$ & $\begin{array}{l}- \\
- \\
-\end{array}$ \\
\hline & & Epistat $^{\oplus}$ & $\begin{array}{l}\text { W: } \\
\text { M: } \\
\text { all: }\end{array}$ & $\begin{array}{c}0(0) \\
1(0.8) \\
1(0.3)\end{array}$ & $\begin{array}{c}- \\
8.3 \\
8.3\end{array}$ & $\begin{array}{l}- \\
8 \\
8\end{array}$ & $\begin{array}{c}- \\
5.8 \\
5.8\end{array}$ \\
\hline
\end{tabular}

374 patients with hereditary hemorrhagic telangiectasia (HHT) treated their epistaxis with different types of nasal self-packings (number of women (W): 215/ 338, 64\%, number of men (M): 123/338, 36\%; gender was not documented in all cases). 129 patients had not used nasal packings, 320 patients used tissues, 120 patients non-pneumatic nasal packings (NPNP) and 46 patients also applied pneumatic nasal packings (PNP); multiple selections within NPNP were possible. The average hemoglobin level (hb, measured in $\mathrm{g} / \mathrm{dl}$ ) of 298 patients and the average number of blood transfusions (BT) of 196 patients with HHT were documented. 299 patients answered the Epistaxis Severity Score (ESS). N = Number of patients with HHT, \% = percentage of (female/ male) patients with HHT who answered the question what kind of nasal self-packing they used, $m=$ mean, SD = standard deviation.

packing is effective, comfortable, secure, and should be easy to insert and remove. Although some data exist on different epis- taxis managements ${ }^{(20)}$, there are no studies that have compared outcomes of different nasal packing techniques. To our know- 
Table 3. Complications of different types of nasal packings in patients with HHT.

\begin{tabular}{|c|c|c|c|c|c|c|}
\hline complications & $\operatorname{sex}$ & $\begin{array}{c}\text { NPNP } \\
(N=17)\end{array}$ & $\begin{array}{c}\text { PNP } \\
(N=17)\end{array}$ & $\begin{array}{l}\text { Tissues } \\
(\mathbf{N}=37)\end{array}$ & $\chi^{2}$ & $\mathbf{p}$ \\
\hline \multirow{3}{*}{ swallowing } & W & $1(14.3)$ & $0(0)$ & $1(5.3)$ & & \\
\hline & $M$ & $3(33.3)$ & $0(0)$ & $1(8.3)$ & & \\
\hline & all & $5(29.4)$ & $0(0)$ & $4(10.8)$ & 6.9 & * \\
\hline \multirow{3}{*}{$\begin{array}{l}\text { hypoxia / } \\
\text { choking fit }\end{array}$} & W & $0(0)$ & $3(37.5)$ & $4(21.1)$ & & \\
\hline & M & $2(22.2)$ & $2(25.0)$ & $2(16.7)$ & & \\
\hline & all & $2(11.8)$ & $5(29.4)$ & $8(21.6)$ & 1.6 & n.s. \\
\hline \multirow{3}{*}{$\begin{array}{l}\text { bleeding did not } \\
\text { stop }\end{array}$} & W & $3(42.9)$ & $2(25.0)$ & $8(42.1)$ & & \\
\hline & M & $2(22.2)$ & $3(37.5)$ & $4(33.3)$ & & \\
\hline & all & $5(29.4)$ & $5(29.4)$ & $14(37.8)$ & 0.6 & n.s. \\
\hline \multirow{3}{*}{$\begin{array}{l}\text { difficulties } \\
\text { removing the } \\
\text { packing }\end{array}$} & W & $2(28.6)$ & $1(12.5)$ & $4(21.1)$ & & \\
\hline & M & $1(11.1)$ & $1(12.5)$ & $4(33.3)$ & & \\
\hline & all & $3(17.6)$ & $2(11.8)$ & $8(21.6)$ & 0.8 & n.s. \\
\hline \multirow{3}{*}{ pain } & W & $0(0)$ & $1(12.5)$ & $0(0)$ & & \\
\hline & $M$ & $1(11.1)$ & $2(25.0)$ & $0(0)$ & & \\
\hline & all & $1(5,9)$ & $3(17,6)$ & $0(0)$ & 6.8 & $*$ \\
\hline \multirow{3}{*}{ infection } & W & $0(0)$ & $1(12.5)$ & $2(10.5)$ & & \\
\hline & M & $0(0)$ & $0(0)$ & $1(8.3)$ & & \\
\hline & all & $0(0)$ & $2(11.8)$ & $3(8.1)$ & 1.9 & n.s. \\
\hline \multirow{3}{*}{ skin irritation } & W & $1(14.3)$ & $0(0)$ & $0(0)$ & & \\
\hline & M & $0(0)$ & $0(0)$ & $0(0)$ & & \\
\hline & all & $1(5.9)$ & $0(0)$ & $0(0)$ & 3.2 & n.s. \\
\hline
\end{tabular}

86 patients with hereditary hemorrhagic telangiectasia $(\mathrm{HHT})(\mathrm{N}=$ $86 / 347,24.8 \%$ ) reported complications or difficulties when applying nasal self-packings; 31 ( $N=31 / 312,9.9 \%)$ knew other patients with HHT who experienced difficulties or complications of any kind. NPNP = non-pneumatic nasal packings, PNP = pneumatic nasal packings, data is presented in number of patients and percentage $(\mathrm{N}(\%)), \mathrm{W}=$ women, $\mathrm{M}$ $=$ men, $x 2=$ chi square, $p=p$-value, ${ }^{*}: p \leq 0.05$, n.s. = not significant.

ledge, this is the first study dealing with the topic of self-packing for nosebleeds and the first to assess 13 different medical nasal packings.

\section{Clinical presentation and treatment of epistaxis}

In our cohort, men consulted their practitioners more frequently than women. In general, women seem to be more likely to contact their practitioners e.g. for gynecological reasons ${ }^{(21-23)}$. Physical symptoms were more important in men whereas women were especially influenced by psychological distress ${ }^{(22)}$. As HHT is an autosomal dominant disorder ${ }^{(18)}$ male and female patients should theoretically be equally affected. A potential reason why more men than women sought help from their practitioner might be due to more severe physical impairments of recurrent epistaxis resulting in iron deficiency anemia ${ }^{(24)}$ or other potentially lethal complications ${ }^{(7)}$. Using a multivariate model, consultation rates were associated with the ESS. Therefore, increasing epistaxis and factors associated with it lead to higher consultation rates, which is easily understandable.

\section{Nasal self-packing}

We attempted to reach patients mainly through patients' advocacy groups. Patients with chronic diseases such as HHT are usually well-educated about their condition and regularly share information amongst themselves and other members of their advocacy groups ${ }^{(25)}$. Nasal self-packing has been routinely demonstrated by otorhinolaryngologists during the annual meetings of the German patient advocacy group for the past ten years. We expected patients to be familiar with the procedure, and to probably use nasal self-packing more often than reported in this study. Some medical knowledge and manual skills might be necessary to insert nasal self-packings. Only $46 \%$ of the patients in our study were taught medical nasal selfpacking technique by their otorhinolaryngologist. Severity of nosebleeds caused about one quarter of patients to use nasal packings regularly. Almost half of the respondents treated themselves only with tissues, probably due to the widespread availability and low costs.

Table 4. Glasgow Benefit Inventory scores for different types of nasal packings.

\begin{tabular}{|c|c|c|c|c|c|c|c|c|}
\hline \multirow[t]{2}{*}{ GBI } & \multicolumn{2}{|c|}{ Tissues } & \multicolumn{2}{|c|}{ NPNP } & \multicolumn{2}{|c|}{ PNP } & \multirow[t]{2}{*}{ F-value } & \multirow[t]{2}{*}{$\mathbf{p}$} \\
\hline & $N(\%)$ & {$[\mathrm{m} \pm \mathrm{SD}]$} & N (\%) & {$[\mathrm{m} \pm \mathrm{SD}]$} & $\mathbf{N}(\%)$ & {$[\mathrm{m} \pm \mathrm{SD}]$} & & \\
\hline total score & \multirow{4}{*}{$137(59)$} & $0.7 \pm 17.1$ & \multirow{4}{*}{$58(25)$} & $7.7 \pm 17.3$ & \multirow{4}{*}{39 (17) } & $10.8 \pm 15.0$ & 6.981 & 0.001 \\
\hline $\begin{array}{l}\text { general } \\
\text { subscale }\end{array}$ & & $0,6 \pm 22.0$ & & $10.3 \pm 23.3$ & & $14.6 \pm 20.7$ & 7.747 & 0.001 \\
\hline $\begin{array}{l}\text { social sub- } \\
\text { scale }\end{array}$ & & $3.6 \pm 14.5$ & & $1.4 \pm 15.7$ & & $3.0 \pm 12.0$ & 0.495 & 0.610 \\
\hline $\begin{array}{l}\text { physical } \\
\text { subscale }\end{array}$ & & $-2.0 \pm 19.0$ & & $3.2 \pm 14.1$ & & $3.4 \pm 14.4$ & 2.734 & 0.067 \\
\hline
\end{tabular}

242 patients with hereditary hemorrhagic telangiectasia (HHT) using different types of nasal packings answered the Glasgow Benefit Inventory (GBI). $\mathrm{NPNP}=$ non-pneumatic nasal packings, $\mathrm{PNP}=$ pneumatic nasal packings, $\mathrm{m}=$ mean, $\mathrm{SD}=$ standard deviation, to compare the GBI scores for the different types of nasal packings a one-way ANOVA was used ( $F$-value), $p=p$-value. 


\section{Complications of nasal self-packings}

Most patients eventually stopped their bleeding sufficiently. However, the main complaint continued to be prolonged nosebleeds. As far as we know, this is the first study of patients evaluating themselves using nasal packings. Patients with recurrent epistaxis might not be able to stop all episodes of nosebleeds sufficiently through nasal self-packing and therefore may have to seek professional help resulting in hospitalizations (26). Experienced physicians in another study were able to insert the appropriate type of packing and therefore stopped the bleeding promptly ${ }^{(27)}$.

In our cohort, NPNP and/ or tissues were more likely to be swallowed. A few cases of life-threatening aspiration of NPNP are described in the literature ${ }^{(28,29)}$. Swallowing of slippery PNP was reported in one case report ${ }^{(27)}$, and subsequently swallow guard butterflies were designed. In our cohort, no cases of swallowed PNP were documented but 5 patients using PNP reported having hypoxia and chocking fits. We addressed this issue during the annual German patient meeting. It is possible that these patients might have been referring to the use of PNP before the swallow guard butterfly was introduced. Apart from this, nasal packings with PNP actually seem to be safe, can be applied by the patients themselves and may prevent hospitalization and related costs. As a result of the applied pressure, patients using PNP complained significantly more often of pain compared to those using NPNP or tissues. A possible explanation might be that the pressure applied by PNP is higher causing more pain. On the contrary, in another study comparing PNP and NPNP (pneumatic low pressure nasal packings (Rapid Rhino ${ }^{\oplus}$ ) vs. Merocel $^{\circledast}$ ), PNP were reported to be less painful. In these studies, physicians inserted nasal packings in patients having had a septoplasty in full anesthesia ${ }^{(19)}$ (this reference is not about repeated nasal packing and is therefore of limited relevance).

Toxic shock syndrome has been described as a potential lethal complication of nasal packing ${ }^{(30-32)}$. The prophylactic use of antibiotics in patients with nasal packings remains controversial ${ }^{(33,34)}$. In our study, less than a tenth of the patients using nasal packings had an infection, and this was similar to other studies ${ }^{(33,35)}$. There might be a bias because patients that have experienced a lethal toxic-shock-syndrome would not have been able to report this anymore. It is also possible that patients found nasal packing uncomfortable and removed them early enough to prevent infections.

\section{Effectiveness and quality of life}

All patients in our cohort reported that their nosebleeds stopped after a while. Heavy and long lasting nosebleeds can make patients feel helpless and therefore panic, resulting in low quality of life ${ }^{(9)}$, Comparing the different types of nasal packing, patients with PNP had the feeling of losing control of a situation less often and had the highest quality of life. Patients using me- dical packings (NPNP and PNP) showed a positive score in every (sub-) score of the Glasgow Benefit Inventory. They reported an improvement in their general daily life, felt healthier and more secure during social events, and were more self-condfident and independent. Tissues used as nasal packings are widely available. This might be the reason why patients applying them reported the greatest benefit regarding their social activities. Patients with PNP included patients using PNP alone and those inserting both PNP and NPNP. In these patients, more options for nasal packings might be associated with more effective packing resulting in a lower blood loss and better physical outcome.

\section{Study limitations}

$\mathrm{HHT}$ is a rare disease making it difficult to gather sufficient numbers of participants for studies at a single treatment center. There are known methodological limitations of survey-based online studies. However, they provide high quantities of data of patients from different countries. Our questionnaire was designed in English and German, and most respondents came from North America and Western Europe (especially Germanand English-speaking countries). Due to language barriers, patients with HHT speaking other languages may not have been reached. Not all patients answered all questions wich led to smaller subgroups for the analysis.

To identify patients suffering from HHT, the modified Curaçao criteria ${ }^{(17)}$ including information about epistaxis was used. Patients with a less severe phenotype (e.g. without epistaxis) might have been missed, resulting in a potential disadvantage. It is likely that we may have collected data from patients who routinely received information on $\mathrm{HHT}$, and have a general interest in the disease as well as some level of acceptance of their condition. This might add to the bias. This selective sampling may explain the higher prevalence of visceral manifestations in our sample (Table 1) compared to reports from other studies. There are no standardized classifications of nasal packings and it was a challenge to categorize them in this study. Most other studies have analyzed just two to three medical nasal packings $(20,36)$, compared to the 13 different medical nasal packings considered in this study. We decided to classify patients with HHT into those inserting PNPs, NPNPs and tissues. Another study (20) compared dissolvable and non-dissolvable nasal packings. As most patients mentioned more than one type of nasal packing, we could not apply this classification. The choice of classification may have had an impact on the subsequent analyses.

The distribution of different types of nasal packings may be due to differences in costs in each country. Not everyone might have had access to all types of nasal packings. Although general gastrointestinal involvement was documented, specific complications leading to blood loss such as gastrointestinal bleedings, the intake of anticoagulants and coagulopathies were not documented. 


\section{Conclusions}

In conclusion, our study showed that most patients with HHT using nasal self-packings could stop the bleeding after a while. Nasal self-packing is a user-friendly and secure method leaving patients more self-confident and independent. Treating physicians should explain and perscribe nasal self-packings and with the support of national self-help groups, patients with HHT should be encouraged to use nasal self-packings in order to improve their quality of life.

\section{Acknowledgements}

The authors are grateful both to the patients with HHT for answering the questionnaire and the advocacy groups (especially HHTCure, Morbus Osler-Selbsthilfe e.V., HHT Swiss, Osler.DK, Telangiectasia Self Help group, and HHTIreland) for their help with distributing the survey. In addition, the authors thank Mrs. Priscilla Meyer and Mr. Ralf Schmiedel for assisting in developing the English version of the questionnaire.

This research did not receive any specific grant from funding agencies in the public, commercial, or not-for-profit sectors.

\section{Authorship contribution}

$\mathrm{FD}, \mathrm{UG}, \mathrm{KT}, \mathrm{CL}$ conceived the study, designed the trial, and obtained research funding. FD, UG, KT, CL supervised the conduct of the trial and data collection. FD, UG, KT, CL undertook recruitment of participating centers and patients and managed the data, including quality control. FD, KT, BAS, CL, SL, and UG provided statistical advice on study design and analyzed the data; FD and UG chaired the data oversight committee. FD, CL and UG drafted the manuscript, and all authors contributed substantially to its revision. FD takes responsibility for the paper as a whole.

\section{Conflict of interest}

BAS has received research grants, reimbursement of travel expenses, speaking and consulting fees from Sutter Medizintechnik, Fisher\&Paykel Healthcare, Neuwirth Medical Products, Philips Healthcare, Inspire Medical, Atos Medical, ALK Abello and SnooZeal Ltd. The other authors declare that they have no conflict of interest.

\section{References}

1. Shovlin $C L$, Guttmacher $A E$, Buscarini $E$, Faughnan ME, Hyland RH, Westermann CJ, et al. Diagnostic criteria for hereditary hemorrhagic telangiectasia (Rendu-Osler-Weber syndrome). Am J Med Genet. 2000;91(1):667.

2. Cole SG, Begbie ME, Wallace GM, Shovlin CL. A new locus for hereditary haemorrhagic telangiectasia (HHT3) maps to chromosome 5. J Med Genet. 2005:42(7):577-82.

3. Shovlin $\mathrm{CL}$, Hughes JM, Tuddenham EG Temperley I, Perembelon YF, Scott J, et al. A gene for hereditary haemorrhagic telangiectasia maps to chromosome 9q3. Nat Genet. 1994;6(2):205-9.

4. Gallione CJ, Repetto GM, Legius E, Rustgi AK, Schelley SL, Tejpar S, et al. A combined syndrome of juvenile polyposis and hereditary haemorrhagic telangiectasia associated with mutations in MADH4 (SMAD4). Lancet. 2004;363(9412):852-9.

5. MCAllister KA, Grogg KM, Johnson DW, Gallione CJ, Baldwin MA, Jackson CE, et al. Endoglin, a TGF-beta binding protein of endothelial cells, is the gene for hereditary haemorrhagic telangiectasia type 1. Nat Genet. 1994;8(4):345-51.

6. Johnson DW, Berg JN, Gallione CJ McAllister KA, Warner JP, Helmbold EA, et al. A second locus for hereditary hemorrhagic telangiectasia maps to chromosome 12 . Genome Res. 1995;5(1):21-8.

7. Stell PM. Epistaxis. Clin Otolaryngol Allied Sci. 1977;2(3):263-73.

8. Geirdal AO, Dheyauldeen S, BachmannHarildstad G, Heimdal K. Quality of life in patients with hereditary hemorrhagic telangiectasia in Norway: a population based study. Am J Med Genet A 2012;158A(6):1269-78.

9. Geisthoff UW, Heckmann K, D'Amelio R, Grunewald S, Knobber D, Falkai P, et al. Health-related quality of life in hereditary hemorrhagic telangiectasia. Otolaryngol Head Neck Surg. 2007;136(5):726-33; discussion 34-5.

10. Lennox PA, Hitchings AE, Lund VJ, Howard DJ. The SF-36 health status questionnaire in assessing patients with epistaxis secondary to hereditary hemorrhagic telangiectasia. Am J Rhinol. 2005;19(1):71-4.

11. Geisthoff UW, Fiorella ML, Fiorella $R$ Treatment of recurrent epistaxis in HHT. Curr Pharm Des. 2006;12(10):1237-42.

12. Osler W. On a Family Form of Recurring Epistaxis Associated with Multiple Telangiectases of the Skin and Mucous Membranes. Johns Hopkins Hosp. Bull., 12, 333-337, 1901.(b) Dickson, WEC: Certain Intracranial Tumors; Their Variability and Multiplicity. Brit Med Jour. 1901;2.

13. Schlosser RJ. Clinical practice. Epistaxis. N Engl J Med. 2009:360(8):784-9.

14. Hoag JB, Terry P, Mitchell S, Reh D, Merlo CA. An epistaxis severity score for hereditary hemorrhagic telangiectasia. Laryngoscope. 2010;120(4):838-43

15. Robinson K, Gatehouse S, Browning GG. Measuring patient benefit from otorhinolaryngological surgery and therapy. Ann Otol Rhinol Laryngol. 1996;105(6):415-22.

16. Haemoglobin concentrations for the diagnosis of anaemia and assessment of severity. Vitamin and Mineral Nutrition Information System. [Internet]. World Health Organization. 2011 [cited 21.02.2018].

17. Hosman AE, Devlin HL, Silva BM, Shovlin CL. Specific cancer rates may differ in patients with hereditary haemorrhagic telangiectasia compared to controls. Orphanet J Rare Dis. 2013:8:195.

18. Plauchu $H$, de Chadarevian JP, Bideau A, Robert JM. Age-related clinical profile of hereditary hemorrhagic telangiectasia in an epidemiologically recruited population. Am J Med Genet. 1989;32(3):291-7.

19. Hesham A, Ghali A. Rapid Rhino versus Merocel nasal packs in septal surgery. J Laryngol Otol. 2011;125(12):1244-6.

20. Fairbanks DN. Complications of nasal packing. Otolaryngol Head Neck Surg. 1986;94(3):412-5.

21. Donaldson JW, McKeever TM, Hall IP, Hubbard RB, Fogarty AW. The UK prevalence of hereditary haemorrhagic telangiectasia and its association with sex, socioeconomic status and region of residence: a population-based study. Thorax. 2014;69(2):161-7.

22. Kapur N, Hunt I, Lunt M, McBeth J, Creed F, Macfarlane G. Primary care consultation predictors in men and women: a cohort study. Br J Gen Pract. 2005;55(511):108-13.

23. Final report to the NHS Information Centre and Department of Health Trends in consultation rates in general practice 1995/1996 to 2008/2009: Analysis of the QResearch ${ }^{\oplus}$ database. [Internet]. Q Research and the Health and Social Care Information Centre. 2009 [cited 21.2.2018].

24. Pastor Valverde C, Sanchez Manzano D, 
Yague Agueda R, Gil Grande R, Gomez Pellico C. [Hereditary hemorrhagic telangiectasia as a cause of iron-deficiency anemia]. Rev Clin Esp. 1993;193(9):483-4.

25. Embuldeniya $G$, Veinot $P$, Bell $E$, Bell $M$, Nyhof-Young J, Sale JE, et al. The experience and impact of chronic disease peer support interventions: a qualitative synthesis. Patient Educ Couns. 2013;92(1):3-12.

26. Viehweg $T L$, Roberson JB, Hudson JW. Epistaxis: diagnosis and treatment. J Ora Maxillofac Surg. 2006;64(3):511-8.

27. Badran K, Malik TH, Belloso A, Timms MS Randomized controlled trial comparing Merocel and RapidRhino packing in the management of anterior epistaxis. Clin Otolaryngol. 2005;30(4):333-7.

28. Smith S, Smith GE, Olowokure B, Ibbotson S, Foord D, Maguire $H$, et al. Early spread of the 2009 influenza $A(\mathrm{H} 1 \mathrm{~N} 1)$ pandemic in the United Kingdom--use of local syndromic data, May-August 2009. Euro Surveill. 2011;16(3).

29. Koudounarakis E, Chatzakis N, Papadakis
I, Panagiotaki I, Velegrakis G. Nasal packing aspiration in a patient with Alzheimer's disease: a rare complication. Int J Gen Med. 2012:5:643-5.

30. Aeumjaturapat S, Supanakorn $S$, Cutchavaree A. Toxic shock syndrome after anterior-posterior nasal packing. J Med Assoc Thai. 2001;84(3):453-8,

31. Nahass RG, Gocke DJ. Toxic shock syndrome associated with use of a nasal tampon. Am J Med. 1988;84(3 Pt 2):629-31.

32. Mansfield CJ, Peterson MB. Toxic shock syndrome: associated with nasal packing. Clin Pediatr (Phila). 1989;28(10):443-5.

33. Derkay CS, Hirsch BE, Johnson JT, Wagner RL. Posterior nasal packing. Are intravenous antibiotics really necessary? Arch Otolaryngol Head Neck Surg. 1989;115(4):439-41.

34. Pepper C, Lo S, Toma A. Prospective study of the risk of not using prophylactic antibiotics in nasal packing for epistaxis. J Laryngol Otol. 2012;126(3):257-9.

35. Herzon FS. Bacteremia and local infections with nasal packing. Arch Otolaryngol. 1971 Oct;94(4):317-20.

36. Iqbal IZ, Jones GH, Dawe N, Mamais C, Smith ME, Williams RJ, et al. Intranasal packs and haemostatic agents for the management of adult epistaxis: systematic review. J Laryngol Otol. 2017;131(12):1065-92.

\section{Dr. Freya Droege}

Univ.-HNO-Klinik (ORL)

Hufelandstrasse 55

45147 Essen

\section{Germany}

Tel: +49 20172385832

Fax: +49 2017231416

E-mail: freya.droege@uk-essen.de 